\title{
Damage occurrence under dynamic loading for anisotropic strain rate sensitive materials
}

\author{
F. Lauro, B. Bennani, J. Oudin \\ Industrial and Human Automatic Control and \\ Mechanical Engineering Laboratory, Mechanical \\ CNRS Research Unit, MECAMAT, CSMA, \\ University of Valenciennes and Hainaut-Cambrésis, \\ France
}

\author{
X. Ni \\ Pam Systems International S.A., France
}

\begin{abstract}
A damage model is integrated into the explicit finite element framework to predict the damage evolution which occurrs under dynamic loading in the crash or stamping process. This damage model is based on the description of the growth, nucleation and coalescence of the microvoids. The microvoid growth is related to the plastic incompressibility equation. The microvoid nucleation is controlled by either the plastic strain or stress. The microvoid coalescence is described by a specific function. This damage process leads to the progressive loss of the structure stress carrying capacity. The ductile fracture occurs once it has vanished. The model is adapted to take the material behaviour anisotropy and damage anisotropy into account. The sensitivity of the damage evolution under dynamic loadings in the case of porous strain rate sensitive material is analysed using single tensile tests. Static and dynamic tensile tests of a notched specimen are performed. Influences of the strain rate and the shape of specimen on the failure mode and loss of the structure's stress carrying capacity are shown.
\end{abstract}

\section{Introduction}

The finite element simulation of the sheet metal forming process is a good way to predict possible material defects inside the final design.

Empirical criteria, such as thickness or plastic strain limits, are usually used as forming limits (Arrieux [2]; Ronde-Ousteau and Moussy [16]).

However, strain rate sensitive materials show an increase in their stress hardening and a decrease in their plastic strain limit when the plastic strain rate increases under dynamic loading (Albertini et al. [1]; Moshksar [12]). These material effects cannot be modelled using the previous empirical criteria.

To model both damage evolution and material dynamic effect, a fully coupled elasto-viscoplastic damage constitutive model has to be used.

The Gurson damage model, modified by Tvergaard, has been used with success to perform metal forming process and crash simulation for isotropic materials (Gurson [7,8]; Lauro et al. [10]; Bennani et al. [5]).

The damage process of porous material is described in terms of the growth, nucleation and coalescence of microvoids. The growth of the existing microvoids is determined from the plastic incompressibility equation. The nucleation of new microvoids by decohesion at the inclusion-matrix interface depends on the inclusion distribution (Needleman [14]; Chu and Needleman [6]; Tvergaard and Needleman [17]). A Gaussian normal distribution is used. The nucleation can be either controlled by the plastic strain or by the stress (Tvergaard and Needleman [18]; Mathur et al. [11]; Needleman and Tveergard [15]). The coalescence of neighbouring microvoids is modelled by the Tvergaard and Needleman's function which describes the rapid loss of the material's stress carrying capacity. The ductile rupture occurs at the end of the damage process once the structure has lost its stress carrying capacity (Hill [9]; Nagaki et al. [13]).

To perform the sheet metal forming process with an anisotropic material, the previous damage model has been modified by introducing Hill's yield stress instead of von Mises' into the Gurson-Tvergaard potential (Beckeret al. [3]). The dynamic effect on the porosity's evolution has been introduced by controlling the microvoid nucleation by stress. The anisotropic damage has been taken into account by modifying the microvoid shape parameters introduced by Tvergaard in the microvoided potential.

The new damage model for anisotropic microvoided material has been integrated into the three-dimensional, Lagrangian, finite element, explicit, vectorized/multitasked code for non linear dynamic analysis of struc- 
tures, PAM-SOLID ${ }^{\mathrm{TM}}$ in the case of convected coordinate shell elements (Belytschko and Tsay [4]).

The present paper initially describes the constitutive damage model and its numerical integration into the finite element code. The damage prediction under dynamic loading is then analysed using single tensile tests on classical and notched specimens. Finally, the influence of the plastic strain rate on the deformed shape and the loss of the material's stress carrying capacity is illustrated by computing static and dynamic tensile tests.

\section{Constitutive damage model}

The evolution of the micro-structural damage is represented by the current void volume fraction $f$ defined by

$$
f=\frac{V_{\mathrm{a}}-V_{\mathrm{m}}}{V_{\mathrm{a}}},
$$

where $V_{\mathrm{a}}, V_{\mathrm{m}}$ are, respectively, the elementary apparent volume of the material and the corresponding elementary volume of the matrix. At time $t=0, f$ is the initial microvoid volume fraction.

The rate of increase of the microvoid volume fraction is given by

$$
\dot{f}=\dot{f}_{\mathrm{n}}+\dot{f}_{\mathrm{g}}
$$

in which $\dot{f}_{\mathrm{g}}$ and $\dot{f}_{\mathrm{n}}$ are, respectively, the nucleation and growth rate of microvoids.

The rate of increase of the microvoid volume fraction, due to the growth of existing microvoids, is determined on the condition that the matrix material is plastically incompressible. The plastic incompressibility condition leads to the following equation

$$
\dot{f}_{\mathrm{g}}=\frac{V_{\mathrm{M}}-\dot{V}_{\mathrm{A}}}{V_{\mathrm{A}}}=(1-f) \operatorname{tr} \boldsymbol{D}^{\mathrm{p}},
$$

in which $D^{\mathrm{p}}$ is the macroscopic plastic strain rate tensor defined in the case of the associated plasticity by

$$
\boldsymbol{D}^{\mathrm{p}}=\dot{\lambda} \frac{\partial \Omega_{\mathrm{evp}}}{\partial \sigma}
$$

with $\sigma$ the Cauchy stress tensor, $\Omega_{\text {evp }}$ the microvoided material potential and $\dot{\lambda}$ the viscoplastic multiplier.

The rate of increase of the void volume fraction is related to the effective plastic strain rate in the case of a Gaussian distribution of the inclusions or second phase inclusions by

$$
\dot{f}_{\mathrm{n}}=\frac{f_{\mathrm{N}}}{S_{\mathrm{N}} \sqrt{2 \pi}} \mathrm{e}^{-\frac{1}{2}\left(\frac{\varepsilon_{\mathrm{M}}-\varepsilon_{\mathrm{N}}}{S_{\mathrm{N}}}\right)^{2}} \dot{\varepsilon}_{\mathrm{M}}=A_{1} \dot{\varepsilon}_{\mathrm{M}},
$$

where $f_{N}$ is the nucleated microvoid volume fraction consistant with the inclusion volume fraction, $S_{N}$ is the Gaussian standard deviation, $\varepsilon_{N}$ is the nucleated effective plastic strain, $\varepsilon_{M}$ is the effective plastic strain, and $A_{1}$ is interpreted as the volume fraction of inclusions converted per unit plastic strain.

Assuming that the nucleation depends only on the maximum stress transmitted across the inclusionmatrix interface, the nucleation microvoid volume fraction rate is expressed by

$$
\begin{aligned}
\dot{f}_{\mathrm{n}}= & \frac{f_{\mathrm{N}}}{S_{\mathrm{N}} \sigma_{y} \sqrt{2 \pi}} \mathrm{e}^{-\frac{1}{2} \frac{\left(\sigma_{\mathrm{M}}+\sigma_{\mathrm{m}}\right)-\sigma_{\mathrm{N}}}{S_{\mathrm{N}} \sigma_{\mathrm{y}}}} \\
& \times\left(\dot{\sigma}_{\mathrm{M}}+\dot{\sigma}_{\mathrm{m}}\right)=A_{2}\left(\dot{\sigma}_{\mathrm{M}}+\dot{\sigma}_{\mathrm{m}}\right),
\end{aligned}
$$

where $f_{\mathrm{N}}$ is the nucleated microvoid volume fraction consistant with the inclusion volume fraction, $S_{\mathrm{N}}$ is the Gaussian standard deviation, $\sigma_{\mathrm{y}}$ initial yield stress, $\sigma_{\mathrm{N}}$ is the mean stress for nucleation, $\left(\sigma_{\mathrm{M}}+\sigma_{\mathrm{m}}\right)$ is the approximation of the maximum stress transmitted across the inclusion-matrix interface and $A_{2}$ is interpreted as the volume fraction of inclusions converted per unit stress.

The yield surface of the porous elasto-viscoplastic material is described by the Gurson's potential modified by Tvergaard defined by

$$
\begin{aligned}
\Omega_{\mathrm{evp}}= & \frac{\sigma_{\mathrm{ef}}^{2}}{\sigma_{\mathrm{M}}^{2}}+2 q_{1} f^{*} \cosh \left(\frac{3}{2} q_{2} \frac{\sigma_{\mathrm{m}}}{\sigma_{\mathrm{M}}}\right) \\
& -\left(1+q_{3} f^{* 2}\right) \quad \text { with } \sigma_{\mathrm{m}}>0,
\end{aligned}
$$

or

$$
\begin{gathered}
\Omega_{\mathrm{evp}}=\frac{\sigma_{\mathrm{ef}}^{2}}{\sigma_{\mathrm{M}}^{2}}+2 q_{1} f^{*}-\left(1+q_{3} f^{* 2}\right) \\
\text { with } \sigma_{\mathrm{m}} \leqslant 0,
\end{gathered}
$$

in which $q_{1}, q_{2}, q_{3}$ are the material parameters of the Gurson potential, $\sigma_{\mathrm{M}}$ is the elasto-viscoplastic flow stress, $\sigma_{\mathrm{m}}$ is the mean stress, $f^{*}$ is Tvergaard and Needleman's coalescence function and $\sigma_{\mathrm{ef}}$ is Hill's effective stress described by

$$
\begin{gathered}
(H+G)^{2} \sigma_{X X}+(H+F)^{2} \sigma_{Y Y} \\
-2 H \sigma_{X X} \sigma_{Y Y}+2 N \sigma_{X Y}^{2}=1,
\end{gathered}
$$

where $H, G, F$ and $N$ are the material parameters of the anisotropic behaviour law and $\sigma_{i j}$ are the components of the Cauchy stress tensor.

The anisotropic shape of the initial microvoids is described by modification of the original material pa- 
rameters $q_{1}, q_{2}$ and $q_{3}$. The lamination of the metal sheet leads to elliptic microvoids. The parameter $q_{1}$ is also defined by the following equation

$$
q_{1}=q_{1} *+m_{0},
$$

where $q_{1}^{*}$ corresponds to the value of $q_{1}$ for a microvoid with a spherical shape and $m_{0}$ is the initial ellipse excentricity defined by

$$
m_{0}=\frac{a_{0}-b_{0}}{a_{0}+b_{0}},
$$

where $a_{0}$ is the orthogonal halfaxis at main strain direction and $b_{0}$ is the parallel halfaxis one.

The coalescence of neighbouring microvoids which leads to the rapid loss of the stress carrying capacity is described using the specific function $f^{*}$ in the Gurson microvoided material potential as shown previously. The coalescence phenomena occurs for a determined level of microvoid volume fraction as described

$$
\begin{gathered}
f^{*}=f_{\mathrm{c}}+\frac{f_{\mathrm{u}}-f_{\mathrm{c}}}{f_{\mathrm{F}}-f_{\mathrm{c}}}\left(f-f_{\mathrm{c}}\right) \\
\text { when } f>f_{\mathrm{c}},
\end{gathered}
$$

or

$$
f^{*}=f \quad \text { when } \quad f \leqslant f_{\mathrm{c}},
$$

where $f_{\mathrm{c}}$ is the critical microvoid volume fraction at coalescence onset, $f_{\mathrm{F}}$ is the microvoid volume fraction at ductile fracture occurrence which corresponds to the complete loss of stress carrying capacity when the effective stress is equal to zero and $f_{\mathrm{u}}$ is the corresponding value of the coalescence function $f_{\mathrm{u}}=f^{*}\left(f_{\mathrm{F}}\right)$.

The ductile fracture is predicted when the complete loss of the stress carrying capacity is reached and $f$ exceeds the critical value $f_{\mathrm{F}}$.

The effective plastic strain rate $\dot{\varepsilon}_{M}$ is computed from the egality between the plastic power dissipated into the material and the corresponding matrix,

$$
\dot{\varepsilon}_{\mathrm{M}}=\frac{\sigma: \boldsymbol{D}^{\mathrm{p}}}{(1-f) \sigma_{\mathrm{M}}},
$$

in which $\sigma$ is the Cauchy stress tensor, $\sigma_{\mathrm{M}}$ is the elasto-viscoplastic flow stress, and $\boldsymbol{D}^{\mathrm{p}}$ is the macroscopic plastic strain rate tensor.

The viscoplastic multiplier $\dot{\lambda}$ is deduced from the consistency condition $\Omega_{\text {evp }}=0$ and $\dot{\Omega}_{\text {evp }}=0$ leading to solve

$$
\begin{aligned}
\Omega_{\mathrm{evp}}=\dot{\Omega}_{\mathrm{evp}}= & \frac{\partial \Omega_{\mathrm{evp}}}{\partial \sigma}: \dot{\sigma}+\frac{\partial \Omega_{\mathrm{evp}}}{\partial \sigma_{\mathrm{M}}}: \dot{\sigma}_{\mathrm{M}} \\
& +\frac{\partial \Omega_{\mathrm{evp}}}{\partial f}: \dot{f}=0 .
\end{aligned}
$$

The expression of the viscoplastic multiplier is finally determined by

$$
\begin{aligned}
\dot{\lambda}= & \Omega_{\mathrm{evp}}\left\{\frac{\partial \Omega_{\mathrm{evp}}}{\partial \sigma}: \boldsymbol{C}^{\mathrm{e}}: \frac{\partial \Omega_{\mathrm{evp}}}{\partial \sigma}-\frac{\partial \Omega_{\mathrm{evp}}}{\partial \sigma_{\mathrm{M}}} \frac{\partial \sigma_{\mathrm{M}}}{\partial \varepsilon_{\mathrm{M}}} A_{3}\right. \\
& \left.-\frac{\partial \Omega_{\mathrm{evp}}}{\partial f}\left[(1-f) \frac{\partial \Omega_{\mathrm{evp}}}{\partial \sigma}: \boldsymbol{I}+A_{4}\right]\right\}^{-1}
\end{aligned}
$$

with

$$
A_{3}=\frac{\sigma: \frac{\partial \Omega_{\mathrm{evp}}}{\partial \sigma}}{(1-f) \sigma_{\mathrm{M}}}
$$

and

$$
A_{4}=A_{1} A_{3}
$$

with the nucleation controlled by plastic strain, or

$$
A_{4}=A_{2}\left[\frac{\partial \sigma_{\mathrm{M}}}{\partial \varepsilon_{\mathrm{M}}} A_{3}+\left(\boldsymbol{C}^{\mathrm{e}}: \frac{\partial \Omega_{\mathrm{evp}}}{\partial \sigma}\right): \boldsymbol{I}\right]
$$

with the nucleation controlled by stress in which $\boldsymbol{C}^{\mathrm{e}}$ is the isotropic material tensor and $\boldsymbol{I}$ is the second order identity tensor.

\section{Numerical implementation}

The previous constitutive damage model is integrated into the three-dimensional, Lagrangian, finite element, explicit, vectorized/multi-tasked code PAM$\mathrm{CRASH}^{\mathrm{TM}}$ as a new constitutive material law.

The new development is a modification of the stress calculation as described below.

In the previous and following equations, the superscript ' $\mathrm{n}$ ' denotes a time increment $n$ (Fig. 1).

\section{Numerical examples}

\subsection{Uniaxial tensile test}

Uniaxial tensile tests of a thin square unit specimen are performed with porous material under dynamic loading to validate the previous numerical integration and to analyse the damage evolution (Fig. 2).

The porous elasto-viscoplastic material is described by Young's modulus $E=210000 \mathrm{MPa}$ and Poisson's ratio $\nu=0.3$ for the elastic part; by the material viscoplastic flow stress

$$
\sigma_{\mathrm{M}}=\left(\sigma_{\mathrm{y}}+E_{\mathrm{T}} \varepsilon_{\mathrm{M}}\right)\left(1+\left(\frac{\dot{\varepsilon}_{\mathrm{M}}}{D}\right)^{1 / p}\right),
$$




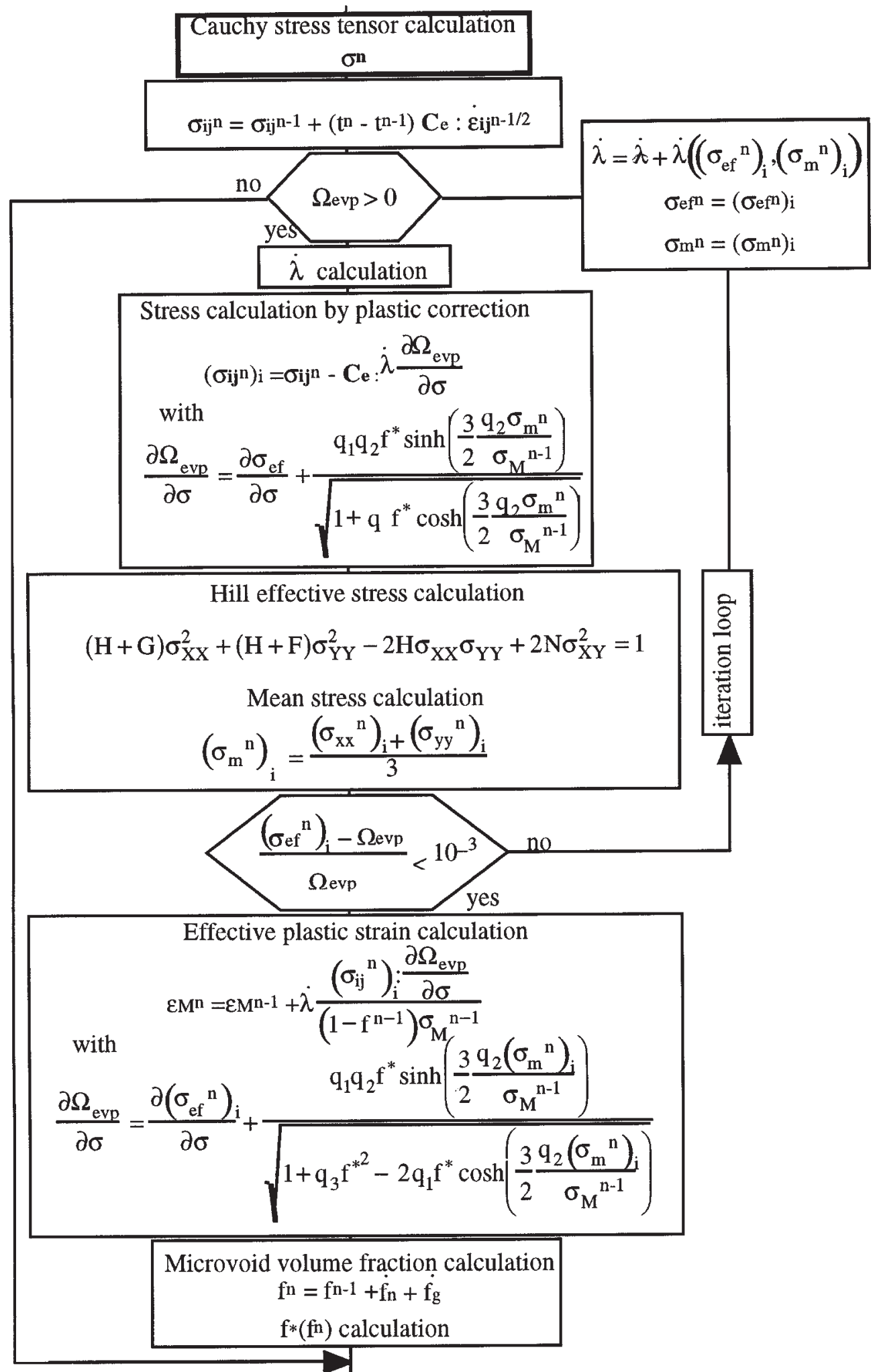

Fig. 1. The modified flowchart of elastic stress prediction and plastic correction algorithm. 
in which the initial yield stress $\sigma_{\mathrm{y}}=200 \mathrm{MPa}$, the tangent modulus $E_{\mathrm{T}}=650 \mathrm{MPa}$ and the dynamic coefficient of the Cowper Symond's law $D=802 \mathrm{~s}^{-1}$ and $p=3.585$ for the viscoplastic part; by the initial microvoided volume fraction $f=0.01$, the material parameters of the microvoided material potential, $q_{1}=1.5, q_{2}=1$ and $q_{3}=2.25$ for the microvoided growth part; by the microvoid volume fraction at coalescence onset $f_{\mathrm{c}}=0.15$ and the microvoid volume fraction at ductile fracture $f_{\mathrm{F}}=0.25$ for the microvoided coalescence part. Both nucleation models in which the nucleation is either controlled by plastic strain or by stress are used to compare their evolutions under dynamic loading. In comparison, the value of the nucleation mean plastic strain $\varepsilon_{N}$ and the nucleation mean stress $\sigma_{\mathrm{N}}$ are defined to give the

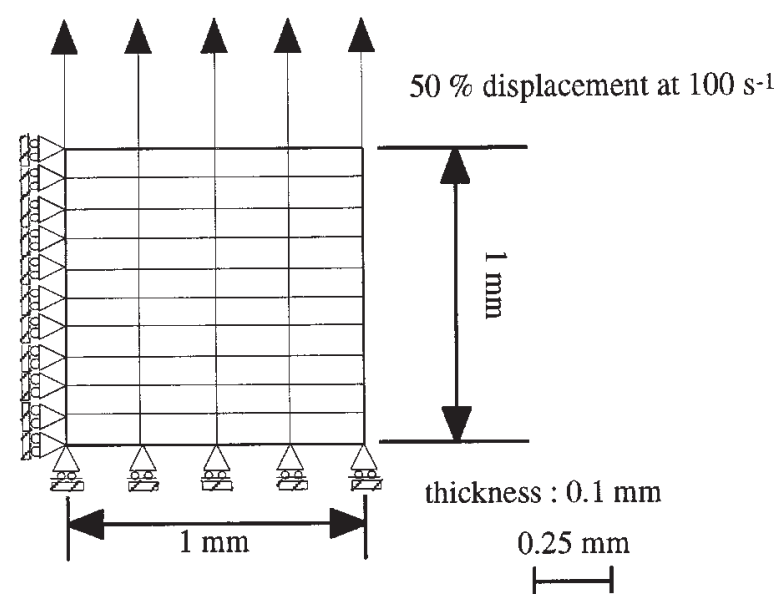

Fig. 2. Uniaxial tensile test, finite element model. same nucleation which increases under static loading. Thus, the nucleation mean plastic strain is 0.2 and the nucleation mean stress is $2.2 \sigma_{\mathrm{y}}$. In both nucleation models, the nucleated microvoid volume fraction consistant with the inclusions volume fraction $f_{\mathrm{N}}$ is equal to 0.04 and the standard deviation $S_{\mathrm{N}}$ is equal to 0.1 . The Hill parameter values $H, F, G$ and $N$ are assumed to have an isotropic behaviour law.

The computations are carried out until $50 \%$ of axial displacement with $100 \mathrm{~s}^{-1}$ constant plastic strain rate. The uniaxial tensile tests are also performed under static loading with porous and pore free material and under dynamic loading with porous and pore free material for comparison. The evolutions of Hill's effective stress in function of the effective plastic strain are shown in Fig. 3 for static and dynamic loading with porous and pore free material in the case of microvoid nucleation controlled by plastic strain or stress.

In both dynamic and static loading, porous material softens. This material softening corresponds to the loss of the stress carrying capacity due to the damage evolution. Under static loading, porous materials with nucleation controlled by plastic strain or stress show the same Hill's effective stress and microvoid volume fraction evolution thereby confirming the previous assumption about the mean nucleation plastic strain and stress values.

However, under dynamic loading, the nucleation prediction and obviously the damage evolution strongly depend on the microvoid nucleation controlled type.

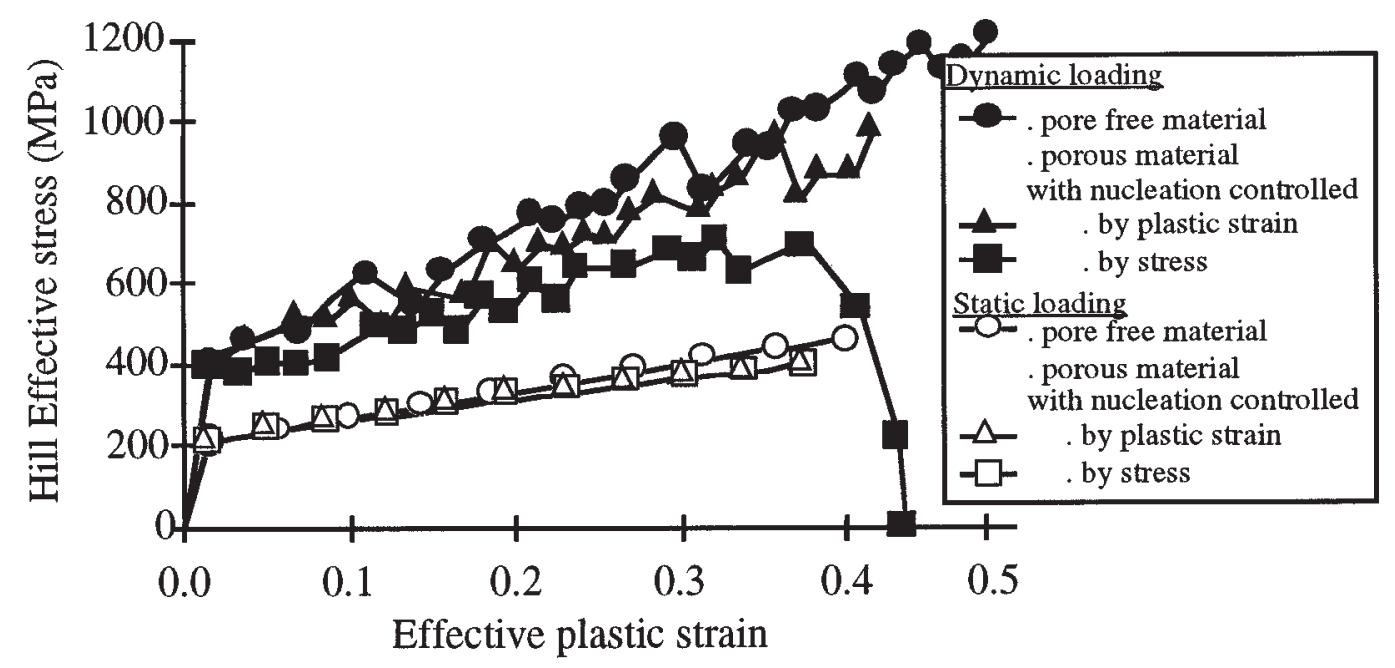

Fig. 3. Uniaxial tensile test, evolution of the effective Hill stress in function of the effective plastic strain. 


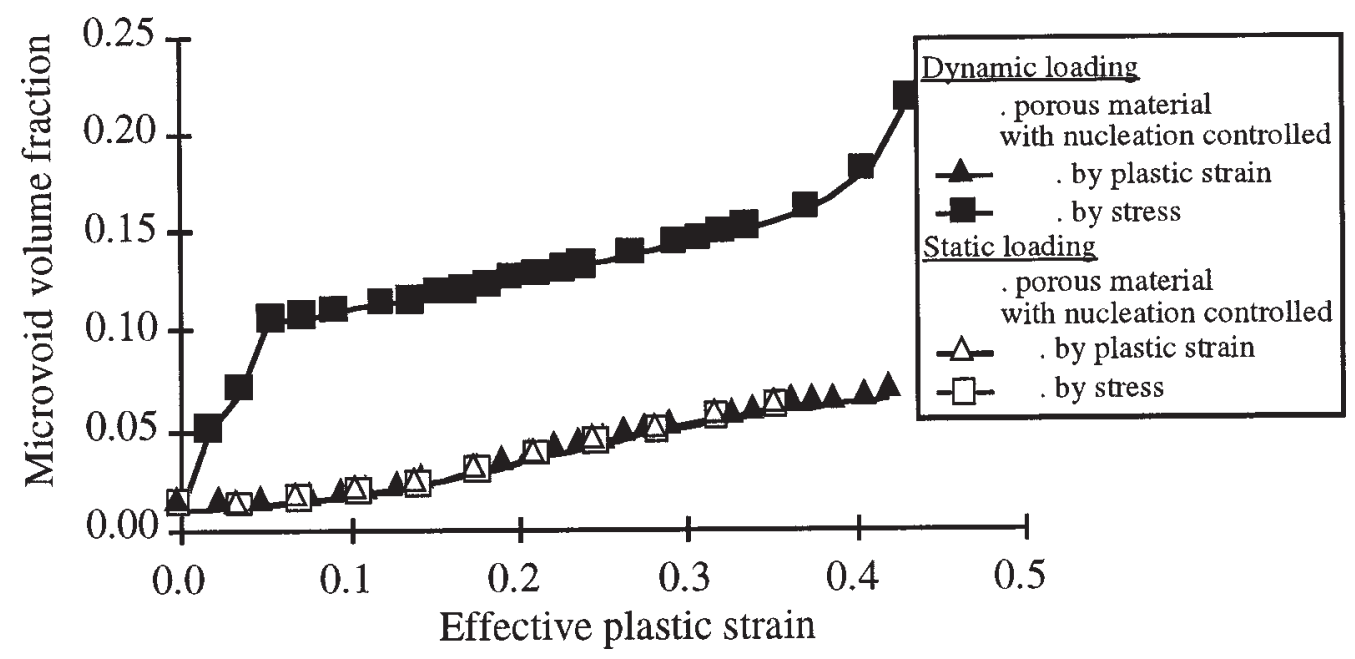

Fig. 4. Uniaxial tensile test, evolution of the microvoid volume fraction in function of the effective plastic strain.

In the case of microvoid nucleation controlled by plastic strain, the damage evolution is independent of the plastic strain rate. Indeed, the microvoid volume fraction evolution in function of the effective plastic strain is the same under static or dynamic loadings. The straining level differs only due to the dynamic correction (Fig. 4).

However, in the case of microvoid nucleation controlled by stress, the microvoided material behaviour is strongly dependent on the plastic strain rate. Due to the dynamic correction, the material matrix quickly reaches the nucleation mean stress value for low plastic strain which leads to the important increase of the microvoid volume fraction shown in Fig. 5. After the nucleation phase, the microvoid volume fraction with microvoid nucleation controlled by stress or plastic strain are similar.

This important increase of the damage evolution leads to the complete loss of the stress carrying capacity and consequently the ductile fracture of the material. This ductile fracture occurs at the 0.43 effective plastic strain. The dynamic loading of the uniaxial tensile test gives rise to the ductile fracture of the material whereas in the case of static loading the microvoid volume fraction does not reach the coalescence onset value.

The uniaxial tensile tests with porous material under dynamic loadings highlight three things: the good numerical implementation of the damage model with elasto-viscoplastic material, the loss of the material's stress carrying capacity with damage evolution and the dependence on the damage evolution with varying plastic strain rates in the case of microvoid nucleation controlled by stress.

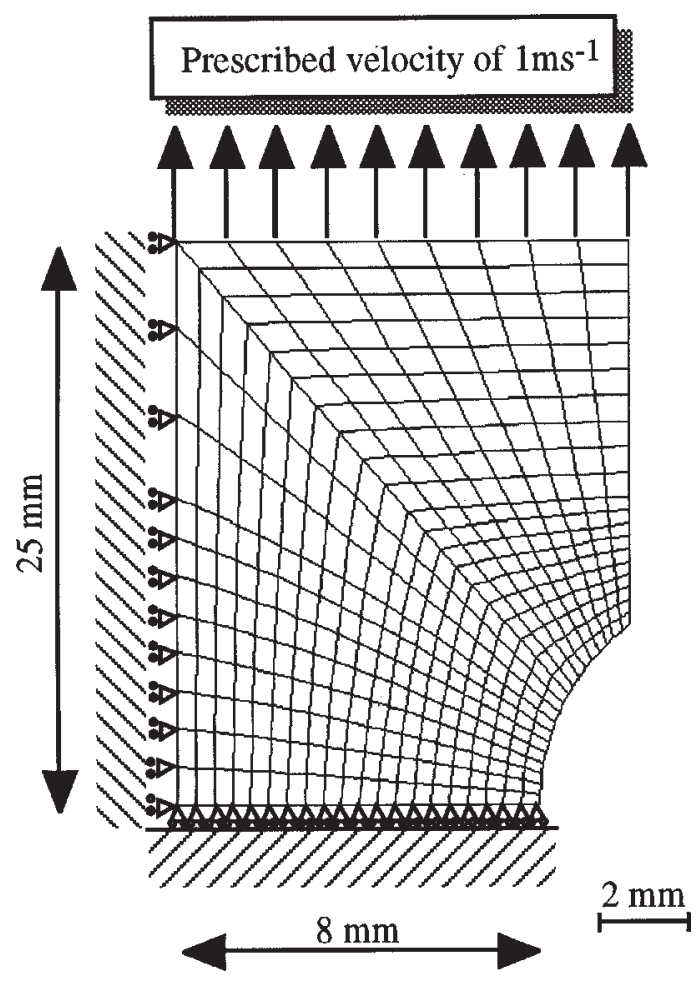

Fig. 5. Finite element mesh of one quarter of the notched specimen.

\subsection{Tensile tests on anisotropic notched specimens}

Uniaxial tensile tests of thin notched specimens are performed with porous material under dynamic loading to analyse the damage evolution. The finite element mesh of the notched specimen is reduced by symetries to one quarter of the notched specimen and is constituted of 400 shell elements (Fig. 5). 


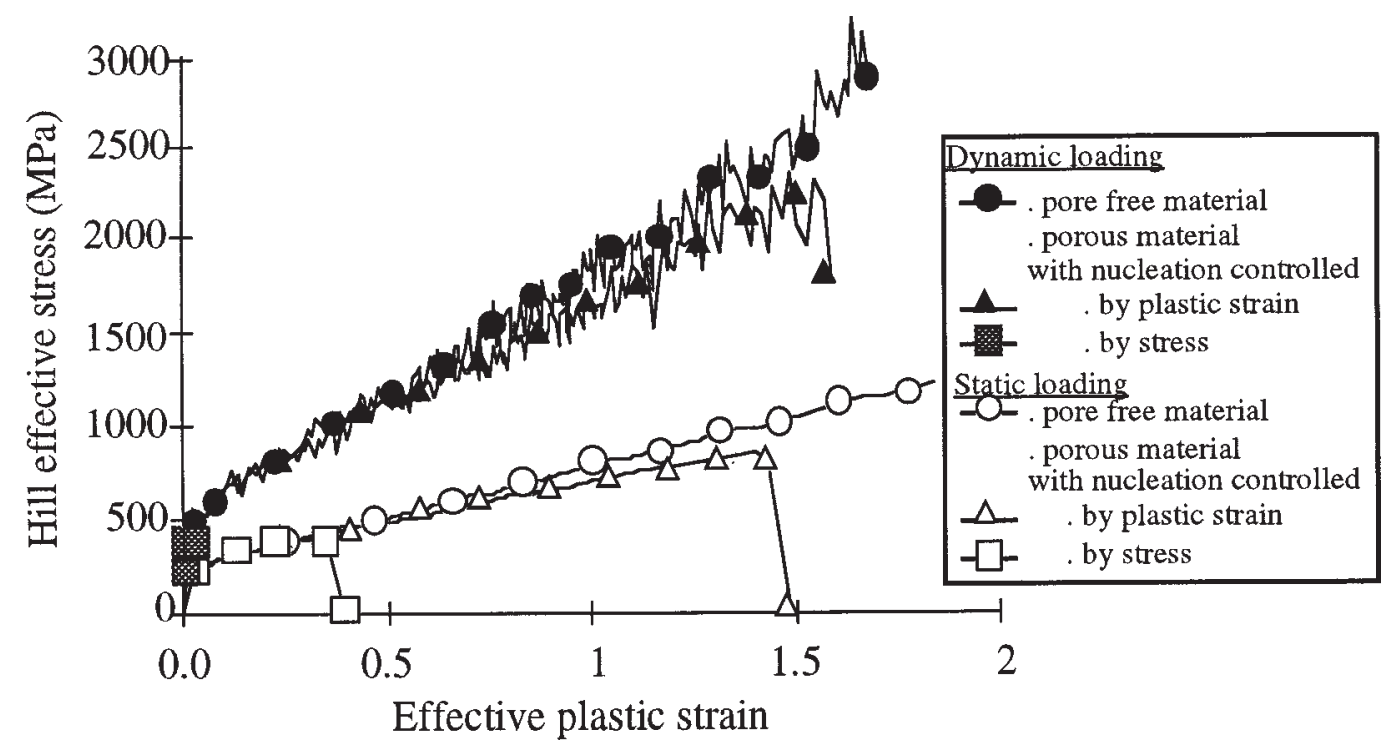

Fig. 6. Uniaxial tensile test, evolution of the effective Hill stress in function of the effective plastic strain.

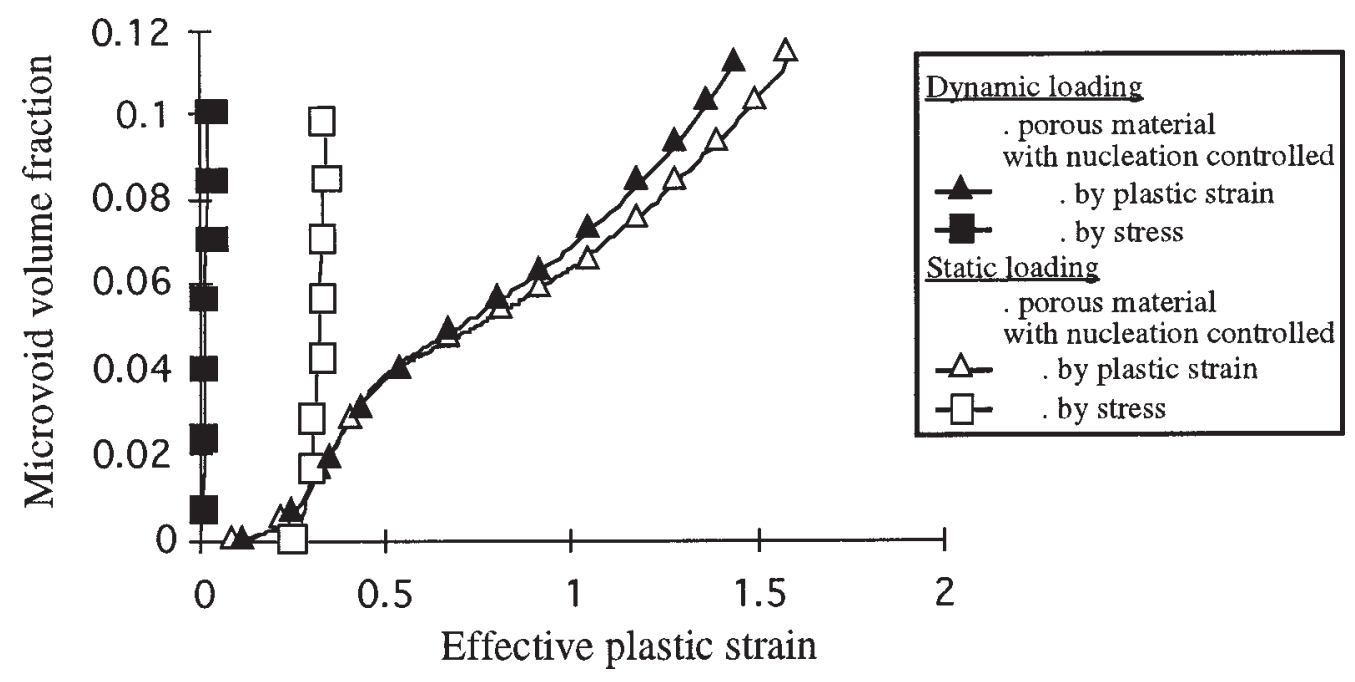

Fig. 7. Uniaxial tensile test, evolution of the microvoid volume fraction in function of the effective plastic strain.

Table 1

Behaviour law of the material

\begin{tabular}{cccccccc}
\hline$E_{\mathrm{t}}$ & 192.5 & 5240 & 1374 & 1184 & 845.8 & 557 & 530 \\
$\sigma_{\mathrm{M}}$ & 225 & 235.5 & 248.9 & 269 & 292.1 & 319.9 & 4000 \\
\hline
\end{tabular}

The material behaviour corresponds to that of a laminated sheet.

The porous elasto-viscoplastic material is described by Young's modulus $E=210000 \mathrm{MPa}$ and Poisson's ratio $\nu=0.3$ for the elastic part; by the material viscoplastic flow stress described in Table 1, with the initial yield stress $\sigma_{\mathrm{y}}=216 \mathrm{MPa}$ and the dynamic coefficient of Cowper Symond's law $D=802 \mathrm{~s}^{-1}$ and $p=3.585$ for the viscoplastic part; by the initial microvoided volume fraction $f=10^{-5}$, the material parameters of the microvoided material potential, $q_{1}=1.295, q_{2}=1.0$ and $q_{3}=1.68$ for the microvoided growth part; by the microvoid volume fraction at coalescence onset $f_{\mathrm{c}}=0.11$ and the microvoid volume fraction at ductile fracture $f_{\mathrm{F}}=0.14$ for the microvoided coalescence part. Both nucleation models in which the nucleation is either controlled by plastic strain or stress are used to compare their evolutions under dynamic loading. In comparison, the value of the nucleation mean plastic strain and the 
nucleation mean stress are defined to give the same nucleation increase under static loading. Thus, the nucleation mean plastic strain is equal to 0.335 and the nucleation mean stress is equal to $2.6 \sigma_{\mathrm{y}}$. In both nucleation models, the nucleated microvoid volume fraction consistent with the inclusions volume fraction $f_{\mathrm{N}}$ is 0.0337 and the standard deviation $S_{\mathrm{N}}$ is 0.1 . The anisotropy is taken into account by introducing Hill's parameter values $H=0.1, F=1.42, G=1.9$ and $N=2.28$ and the rolling direction is considered as the loading direction.

The tensile tests of notched specimens are performed with porous material for static and dynamic

(a)

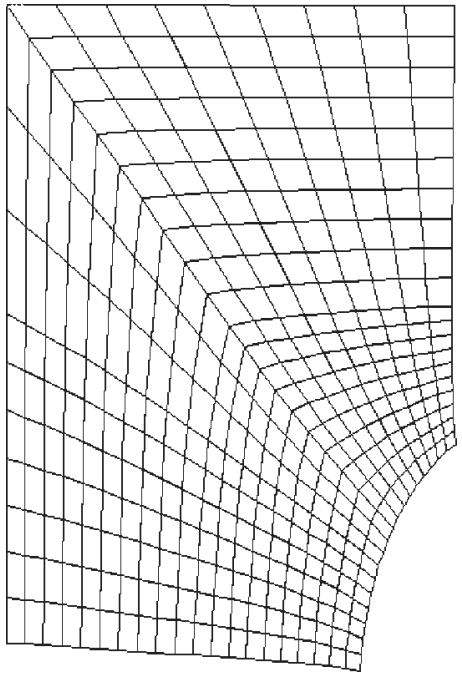

(b)

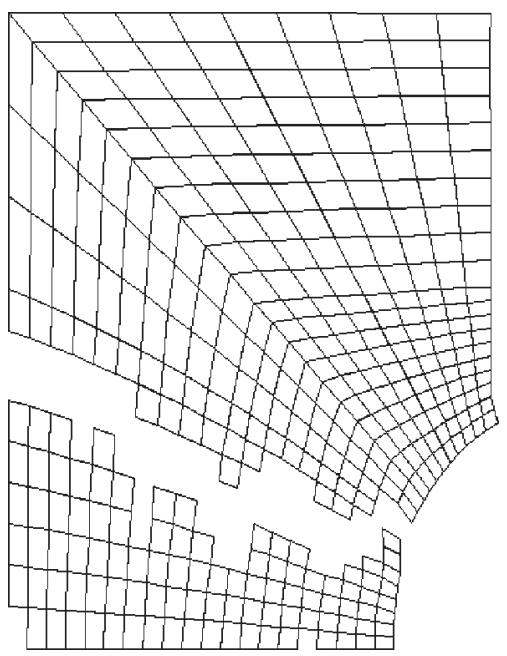

Fig. 8. Failure mode: (a) in the perpendicular plane of the dynamic loading axis for nucleation controlled by plastic strain, (b) in a shear band mode for dynamic loading with nucleation controlled by stress. loadings in the case of nucleation controlled by plastic strain or stress. Computations are also made with undamaged materials for comparison.

As shown in the previous example, the damage evolution is mainly due to the microvoid nucleation. Consequently, the nucleation controlled type directly influences the material's response. In the case of nucleation controlled by plastic strain, evolutions of Hill's effective stress in function of the effective plastic strain are quite similar for static and dynamic loadings (Fig. 6).

Indeed, the microvoid nucleation, independent of the stress distribution, is insensitive to the stress dynamic correction introduced into the CowperSymonds stress law and to the the high stress triaxiality in the notch (Fig. 7).

However, in the case of nucleation controlled by stress, the effects of stress dynamic correction and high stress triaxiality in the notch lead to the rapid loss of the material's stress carrying capacity (Fig. 6). The damage evolution due to microvoid nucleation strongly increases for low effective plastic strain (Fig. 7).

The ductile fracture is predicted for 0.4 and 0.15 effective plastic strain values with static and dynamic loading respectively (Fig. 6).

The failure mode is obviously dependent on the nucleation controlled type. The failure occurs in the plane perpendicular to the loading axis and approximatively in the $45^{\circ}$ shear band with the nucleation controlled by plastic strain and stress for dynamic loading respectively (Fig. 8).

\section{Conclusion}

The Gurson damage model is used to predict the damage evolution occurring under dynamic loading for anisotropic metal sheet by introducing Hill's effective stress in Gurson's potential. This model is based on the description of the material's porosity by the microvoid volume fraction and the prediction of the porous material flow by the Gurson yield surface. The evolution of the microvoid volume fraction, due to the growth of existing microvoids, the nucleation of new microvoids by inclusions fractures or decohesions and the coalescence of neighbouring microvoids, is taken into account. The final ductile rupture is predicted at the complete loss of the stress carrying capacity.

The application of the damage model to simulate uniaxial tensile tests shows that the damage evolution 
depends very much on the nucleation description type. In the case of nucleation controlled by plastic strain the damage evolution is independent on the plastic strain rate. The microvoid volume fraction level is the same under static or dynamic loadings. However, in the case of nucleation controlled by stress, the damage evolution is greatly modified with varying plastic strain rates. The ductile fracture is predicted for low plastic strains at high plastic strain rates.

The tensile tests with porous material illustrate the modification of the microvoid volume fraction distribution and the difference of the failure modes obtained with both nucleation description types.

This paper highlights that

(i) Prediction of the damage evolution in a microstructural way is possible;

(ii) Evolution of the damage during deformation leads to a loss of the resisting capacity of the structure and the softening of the material;

(iii) Damage evolution modifies the local and global responses of the structure under large dynamic loadings;

(iv) Damage evolution and ductile fracture under dynamic loading depend on the nucleation description type.

\section{Acknowledgements}

This work was carried out with the support of the French Ministry of Research and Education, CNRS, Nord Pas de Calais region and FEDER. The provision of PAM-CRASH ${ }^{\mathrm{TM}}$ by ESI Group is gratefully acknowledged. Special thanks to E. Haug and H. Charlier for their technical and financial support. The authors want to thank Thierry Barrière for his help with computational simulations.

\section{References}

[1] C. Albertini, A.M. Eleiche and M. Montagnani, Strain rate history effects on the mechanical properties of AISI 316 stainless steel, in: Metallurgical Applications of Shock Wave and High Strain Rate Phenomena, M.A. Meyers, ed., 1986, pp. 583-602.
[2] R. Arrieux, Determination and use of the forming limit stress diagrams in sheet metal forming, J. Mater. Processes Technol. 53 (1995), 47-56.

[3] R. Becker, R.E. Smelser and O. Richmond, The effect of void shape on the development of damage and fracture in plane strain tension, J. Mech. Phys. Solids 37 (1989), 111-129.

[4] T. Belytschko and C.S. Tsay, Explicit algorithms for the nonlinear dynamics of shells, Computer Methods in Applied Mechanics and Engineering 42 (1984), 225-251.

[5] B. Bennani, P. Picart and J. Oudin, Finite element computation of isotropic and elasto-plastic problems with damage occurence, MECAMAT (1993), 363-369.

[6] C.C. Chu and A. Needleman, Void nucleation effects in biaxially stretched sheets, J. Eng. Mater. Technol. 102 (1980), 249-256.

[7] A.L. Gurson, Porous rigid-plastic materials containing rigid inclusions. Yield function, plastic potential and void nucleation, in: Proc. Int. Fract., Vol. 2A, D.M.R. Taplin, ed., 1977, pp. 357-364.

[8] A.L. Gurson, Continum theory of ductile rupture by void nucleation and growth: Part I. Yield criterion and flow rules for porous materials, J. Eng. Mater. Technol. 2 (1977).

[9] R. Hill, A theory of yielding and plastic flow of anisotropic metals, Proc. R. Soc. 193 (1948), 281-297.

[10] F. Lauro, B. Bennani, P. Drazetic, X. Ni and J. Oudin, Ductile damage and fracture finite element modelling of elastoviscoplastic voided materials, Comput. Mater. Sci. 7 (1997), 295-307.

[11] K.K. Mathur, A. Needleman and V. Tveergard, Ductile failure analyses on massively parallel computers, Comput. Methods Appl. Mech. Eng. 119 (1994), 283-309.

[12] M.M. Moshksar, The effect of strain rate on the mechanical behavior of Al-Si alloy, J. Mater. Proc. Technol. (1993), 383-393.

[13] S. Nagaki, R. Sowerby and M. Goya, An anisotropic yield function for porous metal, Mater. Sci. Eng. A142 (1991), 163-168.

[14] A. Needleman, A continum model for void nucleation by inclusion debonding, J. Appl. Mech. 54 (1987), 525-539.

[15] A. Needleman and V. Tveergard, An analysis of ductile rupture in notched bars, J. Mech. Phys. Solids 32 (1984), 461490.

[16] F. Ronde-Ousteau and F. Moussy, L'emboutissage: Progrès récents et évolutions attendues, MECAMAT (1996), 44-50.

[17] V. Tvergaard and A. Needleman, Analysis of the cup-cone fracture in a round tensile bar, Acta Metall. 32(1) (1984), $157-169$.

[18] V. Tvergaard and A. Needleman, Material strain rate sensitivity in round tensile bar, in: Proc. Cons. Mem. Symp., J. Salençon, ed., 1985, pp. 251-262. 

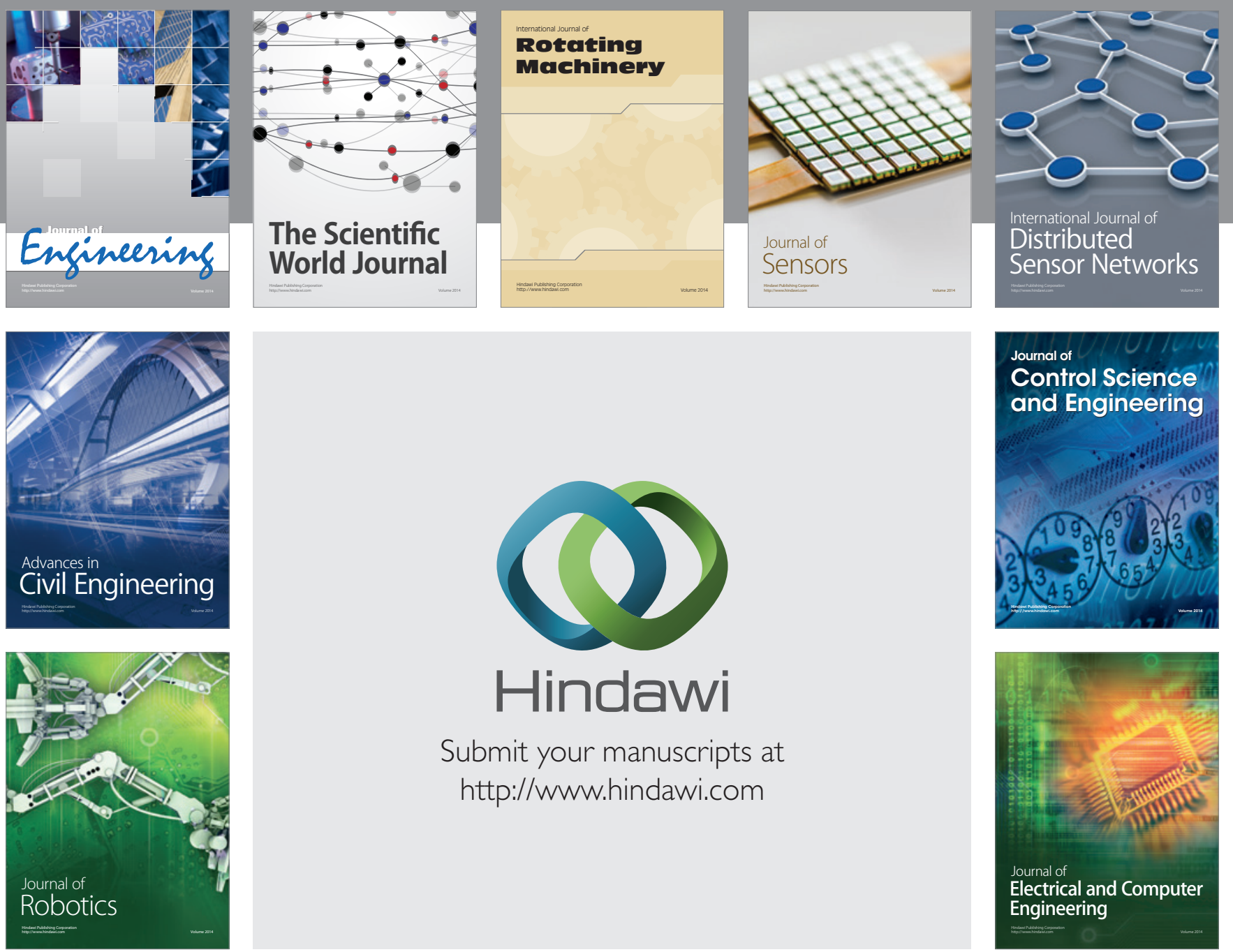

Submit your manuscripts at

http://www.hindawi.com
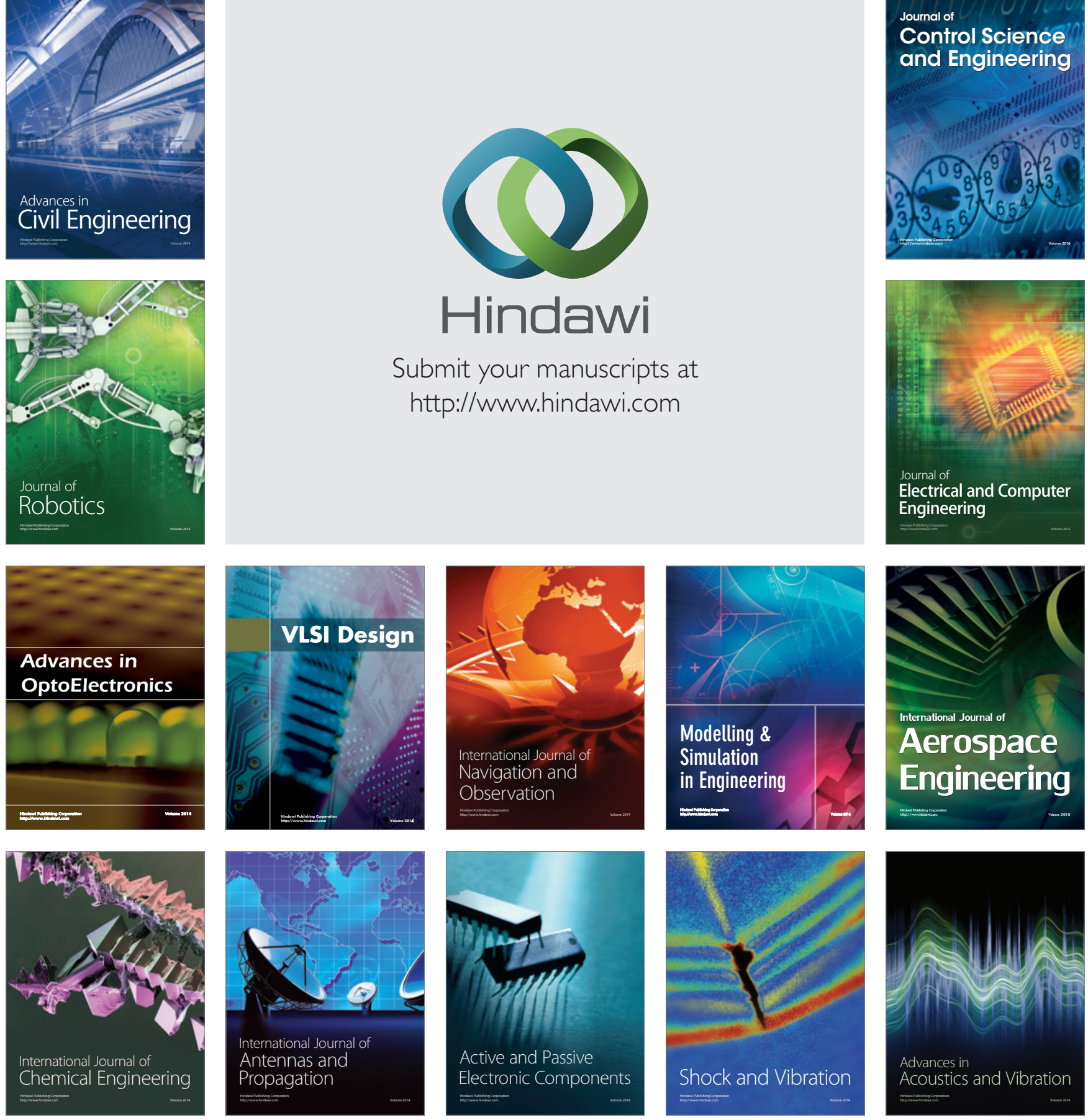